Research Article

\title{
Accurate Integrated Navigation Method Based on Medium Precision Strapdown Inertial Navigation System
}

\author{
Yang Bo $\mathbb{D},{ }^{1}$ Yang Xiaogang $\mathbb{D},{ }^{1}$ Qu Geping, ${ }^{1}$ and Wang Yongjun ${ }^{2}$ \\ ${ }^{1}$ Department of Control Engineering, Xi'an Research Institute of High Technology, Xi'an 710025, China \\ ${ }^{2}$ College of Intelligent Engineering, University of Jinan Quancheng College, Penglai 265600, China
}

Correspondence should be addressed to Yang Bo; yangbo8093@sina.com and Yang Xiaogang; doctoryxg@163.com

Received 17 August 2020; Revised 10 October 2020; Accepted 21 October 2020; Published 9 November 2020

Academic Editor: Gaoge $\mathrm{Hu}$

Copyright $\odot 2020$ Yang Bo et al. This is an open access article distributed under the Creative Commons Attribution License, which permits unrestricted use, distribution, and reproduction in any medium, provided the original work is properly cited.

A method of accurate integrated navigation for high-altitude aerocraft by medium precision strapdown inertial navigation system (SINS), star sensor, and global navigation satellite system (GNSS) is researched in this paper. The system error sources of SINS and star sensor are analyzed and modeled, and then system errors of SINS and star sensor are chosen as system states of integrated navigation. Considering that the output of star sensor is attitude quaternion, it can be regarded as an attitude matrix, then the equivalent attitude matrix is constructed by using the output of SINS, and the calculating equation of the equivalent attitude matrix is designed. Thus, one of the measurements of integrated navigation can be constructed by using the equivalent attitude matrix and the attitude matrix output of star sensor. According to the constraint conditions of the attitude matrix, the diagonal elements are selected as one of the measurements of integrated navigation, and the corresponding measurement equation is derived. At the same time, the velocity output and position output difference between SINS and GNSS is selected as the other measurement, and the corresponding measurement equation is also derived. On this basis, the Kalman filter is used to design an integrated navigation filtering algorithm. Simulation results show that although the medium precision SINS is used, the heading accuracy of this integrated navigation method is better than $\pm 1.5^{\prime}$, the pitch and roll accuracy are better than $\pm 0.9^{\prime}$, the velocity accuracy is better than $\pm 0.05 \mathrm{~m} / \mathrm{s}$, and the position accuracy is better than $\pm 3.8 \mathrm{~m}$. Therefore, the integrated navigation effect is very significant.

\section{Introduction}

For the flight control of high-altitude aerocraft, the navigation ability with high precision and high reliability is very important; especially in the long endurance environment, it is not easy to be affected by external natural environment and human interference. At present, INS (Inertial Navigation System)/satellite-integrated navigation scheme has been widely used in aerospace and other fields, such as INS/GPS (Global Positioning System) integrated navigation [1-4]. However, there are a series of outstanding problems in this navigation mode, such as low heading accuracy, bad estimating effect on the gyro constant drift, and limited antiinterference capability during a long time [5-8]. Particularly, the satellite navigation signals are subject to be interfered with or shielded [8], which will lead to being unable to depend on the satellite navigation system absolutely.
As an advanced attitude measurement instrument, star sensor has the remarkable advantage of high accuracy, strong anti-interference capability, and excellent concealment $[9,10]$. Currently, star sensors are widely used for measuring the attitudes of space crafts accurately [11]. The cost of new style star sensor is getting lower and lower. This has attracted many vendors to the market, and an open literature search shows more and more star tracker vendors offering star sensor at rapidly decreasing costs. Because of its high attitude determination accuracy, star sensor can be used to assist INS/satellite-integrated navigation system to improve its heading accuracy and gyro drift estimation effect and can significantly improve the antijamming ability of the whole integrated navigation system [12]. Particularly with the cost of high-precision star sensor becoming lower and lower, the requirement of INS precision can be significantly reduced by introducing star sensor into the integrated 
navigation system, and the integrated navigation with high precision and high reliability can be realized by using medium precision inertial navigation system.

INS/satellite/star sensor-integrated navigation is studied in [12-14]. In [14], the attitude matrix output by star sensor is used as a measurement, which leads to the complexity of the filtering algorithm and the large amount of filtering calculation. In [12], the attitude angle output by star sensor is used as the measurement, but in fact, the attitude angle of the body relative to geographical coordinate frame cannot be directly output by single star sensor, and its output is the attitude quaternion of the body relative to inertial coordinate frame [15]. In this paper, the attitude quaternion of star sensor is transformed into attitude matrix, and then the equivalent attitude matrix is constructed by using the output of SINS. According to the constraint conditions of attitude matrix, the diagonal elements are selected as one of the measurements of integrated navigation. At the same time, the velocity output and position output difference between SINS and GNSS is selected as the other measurement. As a result, the complexity of integrated navigation filtering algorithm is significantly reduced, and the amount of filtering calculation is reduced.

\section{Accurate Integrated Navigation Scheme}

In the accurate integrated navigation system, east-north-up geographic coordinate frame is taken as the navigation coordinate frame. SINS, star sensor, and GNSS receiver are all assembled on the aerocraft. SINS can output the aerocraft's position, velocity, attitude, and other parameters. The star sensor can output the attitude quaternion $\widetilde{\mathbf{q}}_{i}^{b}$ of the body coordinate frame relative to the inertial coordinate frame. GNSS receiver can output the aerocraft's velocity and position.

Firstly, system errors of SINS and star sensor are chosen as system states of integrated navigation, and then the state equation of integrated navigation is built. Secondly, the equivalent attitude matrix $\hat{\mathbf{C}}_{i}^{b}$ is calculated by using the output of SINS. Thus, the equivalent attitude matrix $\widehat{\mathbf{C}}_{i}^{\mathbb{B}}$ and the attitude quaternion $\widetilde{\mathbf{q}}_{i}^{b}$ of star sensor are used to construct one of the measurements of integrated navigation. At the same time, the velocity output and position output difference between SINS and GNSS is selected as the other measurements. Then all the measurements are sent to the integrated navigation Kalman filter, and the estimations of system states (including system errors of SINS) are obtained by filtering calculating. Finally, the estimations of errors are used to correct the system errors of SINS, and the outputs of the corrected SINS are regarded as the outputs of the integrated navigation system. Accordingly, the structure diagram of this accurate integrated navigation scheme is shown in Figure 1.

During the navigating process, when GNSS receiver is unable to navigate momently for satellite signal being interfered or shielded, the outputs of SINS and star sensor are used to construct measurements for integrated navigation filtering. On the other hand, when star sensor is unable to output the attitude quaternion momently for failing to search star, the outputs of SINS and GNSS receiver are used to construct measurements for integrated navigation filtering. Consequently, this accurate integrated navigation system has strong anti-interference capability and survival capability, which can effectively deal with the interference or destruction.

\section{State Equation of Integrated Navigation System}

In the integrated navigation design, integrated navigation filter is divided into two sorts covering direct method filter and indirect method filter according to the different estimated system state. In the direct method filter, the system state equation of integrated navigation is nonlinear [16], and the extended Kalman filter is usually considered the common method for nonlinear filtering problem. But the calculation of the Jacobian matrices makes the extended Kalman filter difficult to design and implement [4], and the estimating accuracies of parameters are not high. Consequently, the indirect method filter is often adopted to design the integrated navigation system at present. That is, navigation system errors are taken as the system state of integrated navigation, and the optimal estimations of navigation system errors are calculated by Kalman filter or other filters. So the system errors of SINS, star sensor, and GNSS ought to be analyzed and modeled.

For SINS, inertial instrument errors are the main error source of the system, so it is necessary to analyze and model them. Errors of inertial instruments are mainly the random drifts after strict precalibration and compensation, including gyro constant drift, gyro white noise (random walk), accelerometer constant bias, and accelerometer white noise (random walk). Therefore, the error of the gyroscope can be modeled as

$$
\varepsilon_{i}=\varepsilon_{b i}+w_{g i}, \quad(i=x, y, z)
$$

where $\varepsilon_{i}$ represents the errors of the gyroscopes installed along the $x_{-}^{-}, y^{-}$, and $z$-axes of the body, $w_{g i}$ is the white noise of the gyroscope, and $\varepsilon_{b i}$ is the constant drift of the gyroscope that satisfies the following equation:

$$
\dot{\varepsilon}_{b i}=0, \quad(i=x, y, z)
$$

Similarly, the error of the accelerometer can be modeled as

$$
\nabla_{i}=\nabla_{b i}+w_{a i}, \quad(i=x, y, z)
$$

where $\nabla_{i}$ represents the errors of the accelerometers installed along the $x$-, $y$-, and $z$-axes of the body, $w_{a i}$ is the white noise of the accelerometer, and $\nabla_{b i}$ is the constant bias of the accelerometer that satisfies the following equation:

$$
\dot{\nabla}_{b i}=0, \quad(i=x, y, z) .
$$

As a result, the above inertial instrument errors lead to various system errors in SINS, including mathematical platform attitude error, velocity error, and position error. The model equations of these system errors are provided in 


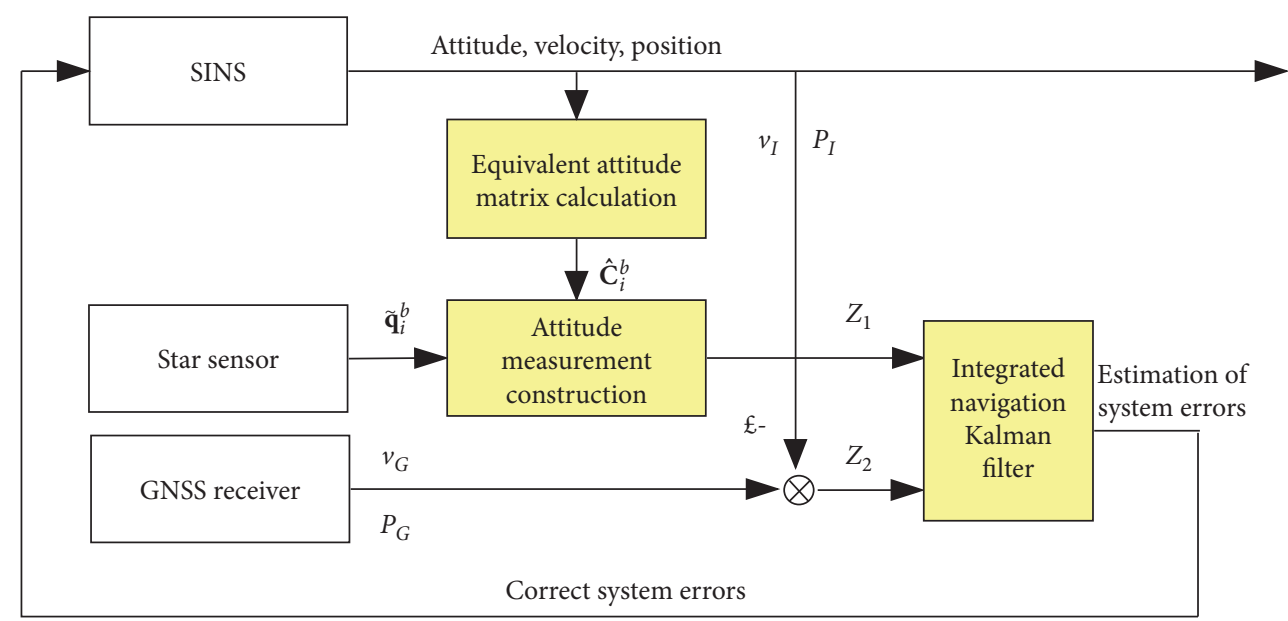

FIGURE 1: Structure diagram of accurate integrated navigation scheme.

some references, so unnecessary details are not given in this paper.

Nowadays, the measurement accuracy of star sensor is very high, and the measurement error is not increasing with time. The attitude measuring accuracy of the CCD (Charge Coupled Device) star sensor has reached the arcsecond range. So the measurement error of star sensor can be regarded as white noise processes. However, the installation error of star sensor is inevitable, which will influence the measuring accuracy of attitude seriously [10]. Therefore, installation error is the most important error source of star sensor, and it is necessary to analyze and model it [17]. Generally, the installation error of star sensor can be considered as a random constant or a first-order Markov process $[10,17]$. In this paper, the random constant is used to describe it; that is,

$$
\delta \dot{A}_{i}=0, \quad(i=x, y, z),
$$

where $\delta A_{i}$ are the installation errors of star sensor relative to the $x^{-}, y^{-}$, and $z$-axes of the body, respectively.

Global navigation satellite system (GNSS) is the most accurate navigation system presently in the world, such as GPS (Global Navigation Satellite System) of USA,
GLONASS (Global Navigation Satellite System) of Russia, and BDS (Bei Dou System) of China. The factors affecting satellite navigation accuracy are more complex, which mainly include ephemeris errors, ionospheric errors, tropospheric errors, transmitting delay errors, and clock errors. Due to the synthetical influence of the above error factors, the three-dimensional position accuracy of satellite navigation usually reaches about decade meters. Due to the fact that satellite navigation accuracy is much higher than other single navigation technologies, the errors of GNSS are directly considered as a white noise process, which are not chosen as system states of integrated navigation anymore. This is useful to reduce the state dimension of the Kalman filter and the computational burden, and thus the computing velocity will be increased.

According to the above analysis, system errors of SINS and star sensor are chosen as system states of integrated navigation, which include SINS mathematical platform attitude errors $\phi_{E}, \phi_{N}, \phi_{U}$, velocity errors $\delta v_{E}, \delta v_{N}, \delta v_{U}$, position errors $\delta L, \delta \lambda, \delta h$, gyro constant drifts $\varepsilon_{b x}, \varepsilon_{b y}, \varepsilon_{b z}$, accelerometer constant biases $\nabla_{b x}, \nabla_{b y}, \nabla_{b z}$, and installation errors of star sensor $\delta A_{x}, \delta A_{y}, \delta A_{z}$. So the state vector $\mathbf{X}$ of integrated navigation system is chosen as follows:

$$
\mathbf{X}=\left[\phi_{E}, \phi_{N}, \phi_{U}, \delta v_{E}, \delta v_{N}, \delta v_{U}, \delta L, \delta \lambda, \delta h, \varepsilon_{b x}, \varepsilon_{b y}, \varepsilon_{b z}, \nabla_{b x}, \nabla_{b y}, \nabla_{b z}, \delta A_{x}, \delta A_{y}, \delta A_{z}\right]^{T}
$$

Based on the state vector $\mathbf{X}$, the system error models mentioned above can be written as follows:

$$
\dot{\mathbf{X}}=\mathbf{F X}+\mathbf{G W}
$$

Equation (7) is the state equation of an accurate integrated navigation system, where $\mathbf{F}$ is the system state matrix, $\mathbf{G}$ is the system noise drive matrix, $\mathbf{W}$ is the system noise matrix, in which $\mathbf{W}=\left[w_{g x}, w_{g y}, w_{g z}, w_{a x}, w_{a y}, w_{a z}\right]^{T}$.

\section{Equivalent Attitude Matrix Calculation}

In the integrated navigation system, outputs of SINS are attitude, velocity, position in geographic coordinate frame, and other parameters, while output of star sensor is the attitude quaternion of body coordinate frame relative to inertial coordinate frame, which is denoted as $\widetilde{\mathbf{q}}_{i}^{b}$. Using the attitude quaternion, the attitude matrix of body coordinate frame relative to inertial coordinate frame can be calculated, 
which is denoted as $\widetilde{\mathbf{C}}_{i}^{b}$. In order to construct the measurement of integrated navigation, it is necessary to calculate the equivalent attitude matrix according to the outputs of SINS and other pieces of information, which is denoted as $\widehat{\mathbf{C}}_{i}^{b}$. This equivalent attitude matrix can match the attitude matrix $\widetilde{\mathbf{C}}_{i}^{b}$ of star sensor.

Suppose the real attitude matrix of the body is $\mathbf{C}_{n}^{b}$ and the real position matrix is $\mathbf{C}_{e}^{n}$. Due to the existence of SINS error, there are errors in the attitude matrix and position matrix obtained by using the output of SINS. The attitude matrix and position matrix are denoted as $\widehat{\mathbf{C}}_{n}^{b}$ and $\widehat{\mathbf{C}}_{e}^{n}$, respectively. The attitude matrix $\widehat{\mathbf{C}}_{n}^{b}$ is calculated by the attitude outputs of SINS, and the position matrix $\widehat{\mathbf{C}}_{e}^{n}$ is calculated by the position outputs of SINS. According to Greenwich Time and Earth rate, the direction cosine matrix relating the inertial and Earth coordinate frames $\mathbf{C}_{i}^{e}$ can be calculated. Then the equivalent attitude matrix $\widehat{\mathbf{C}}_{i}^{b}$ can be calculated by the above information as follows:

$$
\widehat{\mathbf{C}}_{i}^{b}=\widehat{\mathbf{C}}_{n}^{b} \widehat{\mathbf{C}}_{e}^{n} \mathbf{C}_{i}^{e} .
$$

Equation (8) is the calculating equation of the equivalent attitude matrix $\widehat{\mathbf{C}}_{i}^{b}$.

\section{Measurement Equation of Integrated Navigation System}

After obtaining the equivalent attitude matrix $\widehat{\mathbf{C}}_{i}^{b}$ from the outputs of SINS, the matrix $\widehat{\mathbf{C}}_{i}^{b}$ and the attitude matrix $\widetilde{\mathbf{C}}_{i}^{b}$ of star sensor can be used to construct one of the integrated navigation measurements. At the same time, the second measurement of integrated navigation is constructed by using the position and velocity outputs of SINS and the corresponding outputs of GNSS.

Suppose

$$
\mathbf{Z}_{C}=\widehat{\mathbf{C}}_{i}^{b}-\widetilde{\mathbf{C}}_{i}^{b} .
$$

By substituting equation (8) into equation (9) and considering the installation error of star sensor, the following result can be obtained:

$$
\mathbf{Z}_{C}=\widehat{\mathbf{C}}_{n}^{b} \widehat{\mathbf{C}}_{e}^{n} \mathbf{C}_{i}^{e}-(\mathbf{I}+[\boldsymbol{\delta} \mathbf{A}]) \mathbf{C}_{i}^{b}-\mathbf{V}_{S},
$$

where $[\delta \mathbf{A}]$ is the installation error matrix of star sensor and $\mathbf{V}_{S}$ is the measurement white noise matrix of star sensor; that is,

$$
[\boldsymbol{\delta} \mathbf{A}]=\left[\begin{array}{ccc}
0 & -\delta A_{z} & \delta A_{y} \\
\delta A_{z} & 0 & -\delta A_{x} \\
-\delta A_{y} & \delta A_{x} & 0
\end{array}\right]
$$

Considering the mathematical platform attitude error and position error of SINS, equation (10) can be changed to

$$
\mathbf{Z}_{C}=\mathbf{C}_{n}^{b}(\mathbf{I}+[\boldsymbol{\Phi} \times])(\mathbf{I}-[\boldsymbol{\delta} \mathbf{P}]) \mathbf{C}_{e}^{n} \mathbf{C}_{i}^{e}-(\mathbf{I}+[\boldsymbol{\delta} \mathbf{A}]) \mathbf{C}_{n}^{b} \mathbf{C}_{e}^{n} \mathbf{C}_{i}^{e}-\mathbf{V}_{S},
$$

where

$$
\begin{aligned}
{[\boldsymbol{\Phi} \times] } & =\left[\begin{array}{ccc}
0 & -\phi_{U} & \phi_{N} \\
\phi_{U} & 0 & -\phi_{E} \\
-\phi_{N} & \phi_{E} & 0
\end{array}\right], \\
{[\boldsymbol{\delta} \mathbf{P}] } & =\left[\begin{array}{ccc}
0 & -\delta \lambda \sin L & \delta \lambda \cos L \\
\delta \lambda \sin L & 0 & \delta L \\
-\delta \lambda \cos L & -\delta L & 0
\end{array}\right] .
\end{aligned}
$$

Expanding the right side of equation (12) and sorting it out, the following equation can be obtained:

$$
\mathbf{Z}_{C}=\mathbf{C}_{n}^{b}[\boldsymbol{\Phi} \times] \mathbf{C}_{e}^{n} \mathbf{C}_{i}^{e}-\mathbf{C}_{n}^{b}[\boldsymbol{\delta} \mathbf{P}] \mathbf{C}_{e}^{n} \mathbf{C}_{i}^{e}-[\boldsymbol{\delta} \mathbf{A}] \mathbf{C}_{n}^{b} \mathbf{C}_{e}^{n} \mathbf{C}_{i}^{e}-\mathbf{V}_{S}
$$

Considering $\mathbf{C}_{e}^{n} \mathbf{C}_{i}^{e}=\mathbf{C}_{i}^{n}$, equation (14) becomes

$$
\mathbf{Z}_{C}=\mathbf{C}_{n}^{b}[\boldsymbol{\Phi} \times] \mathbf{C}_{i}^{n}-\mathbf{C}_{n}^{b}[\boldsymbol{\delta} \mathbf{P}] \mathbf{C}_{i}^{n}-[\boldsymbol{\delta} \mathbf{A}] \mathbf{C}_{i}^{n}-\mathbf{V}_{S}
$$

Since the attitude matrices $\widehat{\mathbf{C}}_{i}^{b}$ and $\widetilde{\mathbf{C}}_{i}^{b}$ are directional cosine matrices with three rows and three columns, suppose $c_{i j}(i, j=1,2,3)$ is the $i$-row, $j$-column element of the attitude matrix, and then the nine elements of each attitude matrix satisfy the following constraints:

$$
\left\{\begin{array}{l}
c_{11}^{2}+c_{21}^{2}+c_{31}^{2}=1, \\
c_{12}^{2}+c_{22}^{2}+c_{32}^{2}=1, \\
c_{13}^{2}+c_{23}^{2}+c_{33}^{2}=1, \\
c_{11} c_{21}+c_{12} c_{22}+c_{13} c_{23}=0, \\
c_{21} c_{31}+c_{22} c_{32}+c_{23} c_{33}=0, \\
c_{11} c_{31}+c_{12} c_{32}+c_{13} c_{33}=0 .
\end{array}\right.
$$

It is not difficult to see that only three of the nine elements in the above attitude matrix are independent of each other. Therefore, when constructing the measurement of integrated navigation, only three elements can be taken from the difference between $\widehat{\mathbf{C}}_{i}^{b}$ and $\widetilde{\mathbf{C}}_{i}^{b}$, and the difference between $\widehat{\mathbf{C}}_{i}^{b}$ and $\widetilde{\mathbf{C}}_{i}^{b}$ is denoted as $\mathbf{Z}_{\mathbf{C}}$. In this paper, the diagonal element of $\mathbf{Z}_{\mathbf{C}}$ is selected as one of the measurements of integrated navigation.

Suppose $R_{i j}, T_{i j}$, and $V_{i j}(i, j=1,2,3)$ are the $i$-row, $j$ column elements of the matrices $\mathbf{C}_{n}^{b}, \mathbf{C}_{i}^{n}$, and $\mathbf{V}_{S}$, respectively, and then expanding the left and right sides of equation (15) and taking diagonal elements, the following equations can be obtained: 


$$
\begin{aligned}
Z_{11}= & \left(T_{21} R_{13}-T_{31} R_{12}\right) \phi_{E}+\left(T_{31} R_{11}-T_{11} R_{13}\right) \phi_{N}+\left(T_{11} R_{12}-T_{21} R_{11}\right) \phi_{U} \\
& +\left(T_{21} R_{13}-T_{31} R_{12}\right) \delta L+\left(T_{21} R_{11} \sin L-T_{11} R_{12} \sin L+T_{11} R_{13} \cos L-T_{31} R_{11} \cos L\right) \delta \lambda-T_{31} \delta A_{y}+T_{21} \delta A_{z}-V_{11}, \\
Z_{22}= & \left(T_{22} R_{23}-T_{32} R_{22}\right) \phi_{E}+\left(T_{32} R_{21}-T_{12} R_{23}\right) \phi_{N}+\left(T_{12} R_{22}-T_{22} R_{21}\right) \phi_{U} \\
& +\left(T_{22} R_{23}-T_{32} R_{22}\right) \delta L+\left(T_{22} R_{21} \sin L-T_{12} R_{22} \sin L+T_{12} R_{23} \cos L-T_{32} R_{21} \cos L\right) \delta \lambda+T_{32} \delta A_{x}-T_{12} \delta A_{z}-V_{22}, \\
Z_{33}= & \left(T_{23} R_{33}-T_{33} R_{32}\right) \phi_{E}+\left(T_{33} R_{31}-T_{13} R_{33}\right) \phi_{N}+\left(T_{13} R_{32}-T_{23} R_{31}\right) \phi_{U} \\
& +\left(T_{23} R_{33}-T_{33} R_{32}\right) \delta L+\left(T_{23} R_{31} \sin L-T_{13} R_{32} \sin L+T_{13} R_{33} \cos L-T_{33} R_{31} \cos L\right) \delta \lambda-T_{23} \delta A_{x}+T_{13} \delta A_{y}-V_{33} .
\end{aligned}
$$

where $Z_{i j}(i, j=1,2,3)$ is the $i$-row, $j$-column element of the matrix $\mathbf{Z}_{\mathrm{C}}$.

Suppose $\mathbf{Z}_{1}=\left[Z_{11}, Z_{22}, Z_{33}\right]^{T}$, and then the column vector $Z_{1}$ is one of the integrated navigation measurements. Thus, according to the state vector equation (6) of the integrated navigation system, the following equation can be obtained:

$$
\mathbf{Z}_{1}=\mathbf{H}_{1} \mathbf{X}-\mathbf{V}_{1}
$$

Equation (18) is one of the integrated navigation measurement equations, where $\mathbf{H}_{1}$ is the measurement matrix and $\mathbf{V}_{1}\left(\mathbf{V}_{1}=\left[V_{11}, V_{22}, V_{33}\right]^{T}\right)$ is the white measurement noise vector of star sensor.

The specific form of the measurement matrix $\mathbf{H}_{1}$ is

$$
\mathbf{H}_{1}=\left[\begin{array}{lllll}
\mathbf{H}_{11} & \mathbf{O}_{3 \times 3} & \mathbf{H}_{12} & \mathbf{O}_{3 \times 6} & \mathbf{H}_{13}
\end{array}\right],
$$

where

$$
\begin{aligned}
\mathbf{H}_{11} & =\left[\begin{array}{lll}
T_{21} R_{13}-T_{31} R_{12} & T_{31} R_{11}-T_{11} R_{13} & T_{11} R_{12}-T_{21} R_{11} \\
T_{22} R_{23}-T_{32} R_{22} & T_{32} R_{21}-T_{12} R_{23} & T_{12} R_{22}-T_{22} R_{21} \\
T_{23} R_{33}-T_{33} R_{32} & T_{33} R_{31}-T_{13} R_{33} & T_{13} R_{32}-T_{23} R_{31}
\end{array}\right], \\
\mathbf{H}_{12} & =\left[\begin{array}{lll}
T_{21} R_{13}-T_{31} R_{12} & T_{21} R_{11} \sin L-T_{11} R_{12} \sin L+T_{11} R_{13} \cos L-T_{31} R_{11} \cos L & 0 \\
T_{22} R_{23}-T_{32} R_{22} & T_{22} R_{21} \sin L-T_{12} R_{22} \sin L+T_{12} R_{23} \cos L-T_{32} R_{21} \cos L & 0 \\
T_{23} R_{33}-T_{33} R_{32} & T_{23} R_{31} \sin L-T_{13} R_{32} \sin L+T_{13} R_{33} \cos L-T_{33} R_{31} \cos L & 0
\end{array}\right], \\
\mathbf{H}_{13}= & {\left[\begin{array}{ccc}
0 & -T_{31} & T_{21} \\
T_{32} & 0 & -T_{12} \\
-T_{23} & T_{13} & 0
\end{array}\right] . }
\end{aligned}
$$

At the same time, the differences between the velocity, position outputs of SINS, and the corresponding outputs of GNSS receiver are taken as the other integrated navigation measurement $\mathbf{Z}_{2}$; that is,

$$
\mathbf{Z}_{2}=\left[v_{\mathrm{EI}}-v_{\mathrm{EG}}, v_{\mathrm{NI}}-v_{\mathrm{NG}}, v_{\mathrm{UI}}-v_{\mathrm{UG}}, L_{I}-L_{G}, \lambda_{I}-\lambda_{G}, h_{I}-h_{G}\right]^{T},
$$

where $v_{\mathrm{EI}}, v_{\mathrm{NI}}, v_{\mathrm{UI}}$ are the velocity outputs of SINS in the east, north, and up directions, respectively; $v_{\mathrm{EG}}, v_{\mathrm{NG}}, v_{\mathrm{UG}}$ are the velocity outputs of GNSS receiver in the east, north, and up directions; $L_{I}, \lambda_{I}, h_{I}$ are the latitude, longitude, and altitude outputs of SINS; and $L_{G}, \lambda_{G}, h_{G}$ are the latitude, longitude, and altitude outputs of GNSS receiver.

Obviously, considering the errors of SINS and GNSS, equation (21) can be changed into

$$
\mathbf{Z}_{2}=\left[\delta v_{E}-\delta v_{\mathrm{EG}}, \delta v_{N}-\delta v_{\mathrm{NG}}, \delta v_{U}-\delta v_{\mathrm{UG}}, \delta L-\delta L_{G}, \delta \lambda-\delta \lambda_{G}, \delta h-\delta h_{G}\right]^{T},
$$

where $\delta v_{\mathrm{EG}}, \delta v_{\mathrm{NG}}, \delta v_{\mathrm{UG}}$ are the velocity errors of GNSS in the east, north, and up directions and $\delta L_{G}, \delta \lambda_{G}, \delta h_{G}$ are the latitude, longitude, and altitude error of GNSS.

Similarly, based on the integrated navigation state vector equation (6) and the measurement vector equation (22), the other measurement equation of integrated navigation can be obtained as follows:

$$
\mathbf{Z}_{2}=\mathbf{H}_{2} \mathbf{X}-\mathbf{V}_{2} \text {, }
$$

where $\mathbf{H}_{2}$ is the measurement matrix and $\mathbf{V}_{2}\left(\mathbf{V}_{2}=\left[\delta v_{\mathrm{EG}}, \delta v_{\mathrm{NG}}, \delta v_{\mathrm{UG}}, \delta L_{G}, \delta \lambda_{G}, \delta h_{G}\right]^{T}\right)$ is the white measurement noise vector of GNSS receiver.

The specific form of the measurement matrix $\mathbf{H}_{2}$ is

$$
\mathbf{H}_{2}=\left[\begin{array}{lll}
\mathbf{O}_{6 \times 3} & \mathbf{I}_{6 \times 6} & \mathbf{O}_{6 \times 9}
\end{array}\right] .
$$

Upon that, according to the above equations (18) and (23), the measurement equation of accurate integrated navigation system can be written as follows: 


$$
\left[\begin{array}{l}
\mathbf{Z}_{1} \\
\mathbf{Z}_{2}
\end{array}\right]=\left[\begin{array}{l}
\mathbf{H}_{1} \\
\mathbf{H}_{2}
\end{array}\right] \mathbf{X}-\left[\begin{array}{l}
\mathbf{V}_{1} \\
\mathbf{V}_{2}
\end{array}\right] .
$$

Based on the state equation (7) and measurement equation (25) of an accurate integrated navigation system, filtering calculation can be carried out by Kalman filtering equation, and then the estimation $\widehat{\mathbf{X}}_{k}(k=1,2,3, \ldots)$ of system state vector at time $k$ is calculated recursively in real time. The system state vector includes errors of SINS and star sensor, so the above estimation of the system state vector is taken to correct errors of SINS in time, and the outputs of the corrected SINS are regarded as the outputs of the accurate integrated navigation system, which include the attitude, velocity, and position.

\section{Simulation Results and Discussion}

Firstly, the flight trajectory of the aerocraft is designed and simulated. It is assumed that the initial position of the aerocraft is $36^{\circ} 18.365^{\prime} \mathrm{N}, 110^{\circ} 32.126^{\prime} \mathrm{E}$, and altitude $1560 \mathrm{~m}$; the initial heading angle is $40^{\circ}$, the pitch angle is $0^{\circ}$, and the roll angle is $0^{\circ}$ (all are set randomly); the aerocraft is stationary at the initial moment and the velocity is zero. In the flight process of the aerocraft, a variety of motion forms are designed, such as accelerated taxiing, climbing, constant velocity level flying, turning, diving, and deceleration landing. The flight trajectory and related parameters of the aerocraft are simulated by MATLAB language. Figure 2 shows the three-dimensional curve of the flight trajectory of the aerocraft.

In order to reduce the system cost-effectively, SINS with medium precision is adopted in the simulation experiment. Suppose gyro constant drift is $0.5^{\circ} / \mathrm{h}$ and gyro random walk is $0.05^{\circ} / \sqrt{h}$; accelerometer constant bias is $5 \times 10^{-4} \mathrm{~g}$ and accelerometer random walk is $5 \times 10^{-5} \mathrm{~g} \cdot \sqrt{\mathrm{s}}$; attitude measuring accuracy of star sensor is $30^{\prime \prime}(1 \sigma)$ and installation errors of star sensor relative to the $x$-, $y$-, and $z$-axes of the body are $2^{\prime},-3^{\prime}$, and $1^{\prime}$, respectively; position accuracy and velocity accuracy of GNSS receiver are $15 \mathrm{~m}(1 \sigma)$ and $0.1 \mathrm{~m} / \mathrm{s}$ $(1 \sigma)$. The initial horizontal attitude error of SINS is $5^{\prime}$ and the initial heading error is $20^{\prime}$; the initial position error is $10 \mathrm{~m}$ and the initial velocity error is $0.1 \mathrm{~m} / \mathrm{s}$. The simulation time is $1800 \mathrm{~s}$ and the Kalman filtering cycle is $1 \mathrm{~s}$.

In order to test the performance of this accurate integrated navigation method, SINS/GNSS-integrated navigation is also simulated in this paper, so that the two navigation methods can be compared. Based on the above simulation conditions and flight trajectory, the simulation results are shown in Figures 3-5, respectively. In the figures, the solid line indicates the simulation result of SINS/GNSS/star sensor accurate integrated navigation, and the dotted line indicates the simulation result of SINS/GNSS-integrated navigation.

The above simulation results show that SINS/GNSS/star sensor-integrated navigation has high navigation accuracy and superior comprehensive performance. Although the medium precision SINS and star sensor are used, the attitude accuracy of SINS/GNSS/star sensor-integrated navigation is better than that of SINS/GNSS. The heading accuracy of the

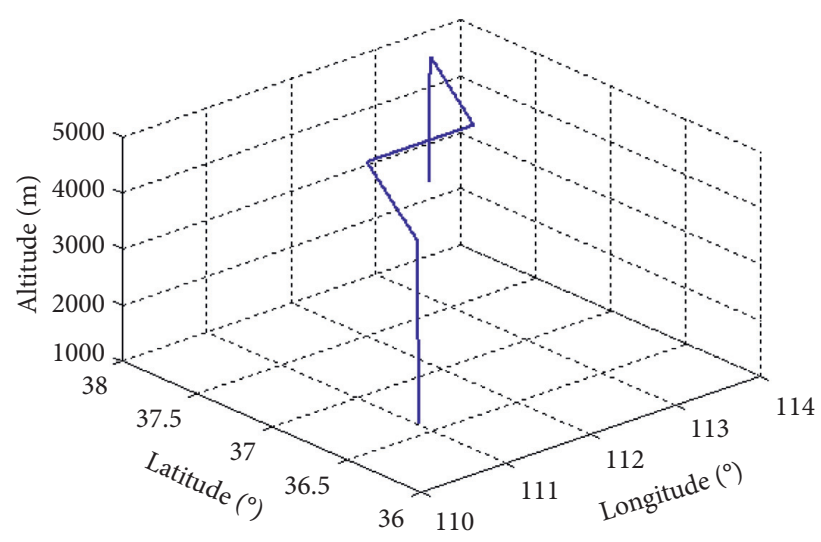

FIgURE 2: Three-dimensional curve of flight trajectory.
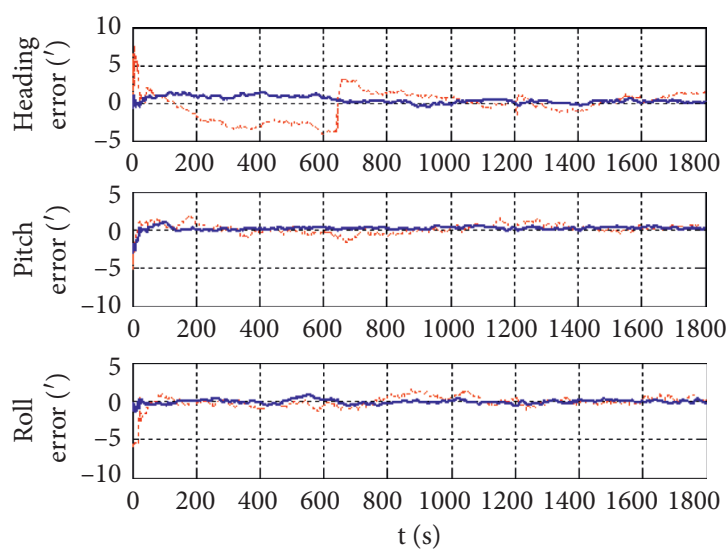

Figure 3: Attitude errors of integrated navigation.

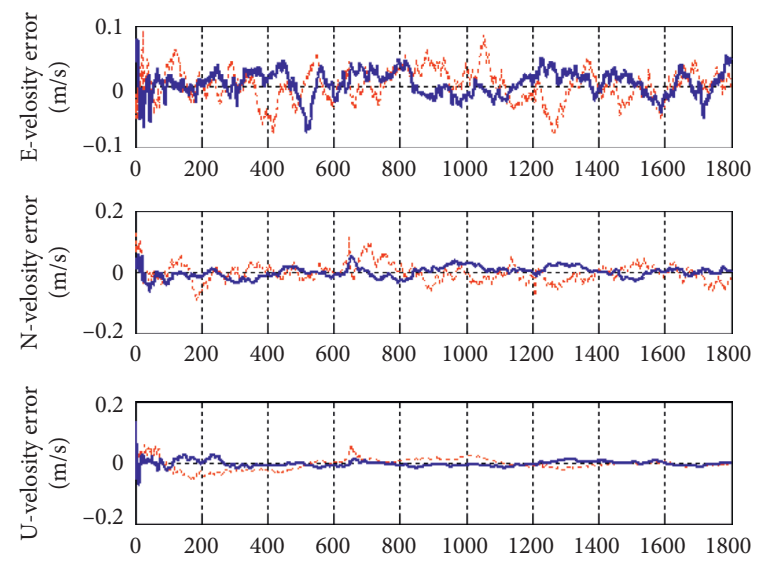

FIGURE 4: Velocity errors of integrated navigation.

former is better than $\pm 1.5^{\prime}$, the pitch and roll accuracy are better than $\pm 0.9^{\prime}$, the heading error of the latter reaches $\pm 4.1^{\prime}$, and the pitch and roll errors reach $\pm 1.7^{\prime}$. It can be seen that after the introduction of star sensor, the heading accuracy of the integrated navigation system is improved obviously. Moreover, for SINS/GNSS/star sensor accurate integrated navigation, the convergence velocities of attitude errors are very quick, and the stable state is achieved in $20 \mathrm{~s}$. 

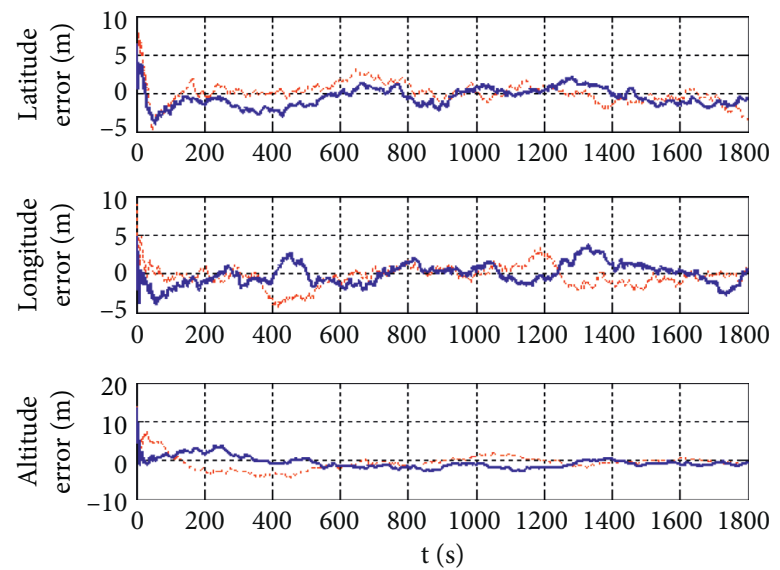

Figure 5: Position errors of integrated navigation.

This is because the high-precision attitude information of star sensor is introduced as measurement and the observability of attitude error of SINS mathematical platform in system state variables is significantly improved; thus, the estimation accuracy and estimation speed of mathematical platform attitude error are improved. Obviously, once the estimation accuracy of the attitude error of the mathematical platform is improved, the accuracy of the heading angle and horizontal attitude angle obtained by using the mathematical platform will be correspondingly improved. Similarly, once the attitude error of the mathematical platform is quickly estimated, the errors of the heading angle and horizontal attitude angle will also converge rapidly. In addition to the attitude accuracy, after the introduction of star sensor, the velocity and position accuracy of the former have been improved to some extent, the horizontal velocity accuracy is kept in $\pm 0.05 \mathrm{~m} / \mathrm{s}$, the vertical velocity accuracy is kept in $\pm 0.03 \mathrm{~m} / \mathrm{s}$, and the position accuracy is kept in $\pm 3.8 \mathrm{~m}$; the horizontal velocity accuracy of the latter is $\pm 0.11 \mathrm{~m} / \mathrm{s}$, the vertical velocity accuracy is $\pm 0.06 \mathrm{~m} / \mathrm{s}$, and the position accuracy is $\pm 4.5 \mathrm{~m}$. The simulation results of the two integrated navigation modes are compared as shown in Table 1.

At the same time, the simulation results also show that the SINS/GNSS/star sensor-integrated navigation filter can not only achieve high navigation accuracy but also better estimate the inertial instrument errors and star sensor installation errors. The estimation results are shown in Figures 6-8.

The above simulation results prove that this accurate integrated navigation method can estimate errors of inertial instruments and installation error of star sensor effectively. In Figure 6, estimating results on constant drifts of gyros in the $x^{-}, y^{-}$, and $z$-axes are excellent, and the estimations are close to the real values of gyro constant drifts $\left(0.5^{\circ} / \mathrm{h}\right)$. It can be seen that over 80 percent of gyro constant drifts have been estimated. It is not difficult to see that the problem of bad estimating effect on the gyro constant drift in INS/satelliteintegrated navigation mode can be effectively solved by introducing star sensor into INS/satellite-integrated navigation system. This is because the introduction of highprecision attitude information of star sensor into measurement can effectively improve the observability of gyro constant drift in system state variables, and then the estimation effect of gyro constant drift is improved. Figure 7 shows that estimating results on constant biases of accelerometers in the $x^{-}, y_{\text {-, }}$, and $z$-axes are very good, and the estimations are very close to the real values of accelerometer constant biases $\left(5 \times 10^{-4} \mathrm{~g}\right)$. It can be seen that about 92 percent of accelerometer constant biases can be estimated. Figure 8 shows that estimating results on installation errors of star sensor are also good, and the estimations are very close to the real values of installation errors $\left(2^{\prime},-3^{\prime}\right.$, and $\left.1^{\prime}\right)$, and over 85 percent of installation errors can be estimated by this accurate integrated navigation method.

In order to test the anti-interference capability of this accurate integrated navigation method, GNSS and star sensor are supposed to be out of order successively in the simulation experiment. Suppose that there are the following instances appearing in the navigation process: (1)GNSS is unable to navigate for satellite signal being interfered from time $400 \mathrm{~s}$ to $600 \mathrm{~s}$ and from time $1100 \mathrm{~s}$ to $1300 \mathrm{~s}$. (2) Star sensor is unable to output the attitude quaternion for failing to search star from time $800 \mathrm{~s}$ to $1000 \mathrm{~s}$ and from time $1400 \mathrm{~s}$ to $1600 \mathrm{~s}$. Based on the above simulation conditions, this accurate integrated navigation method is simulated in this paper, and the main simulation results are given as follows (Figures 9-11).

The above simulation results (Figures 9-11) show that when GNSS or star sensor is out of order successively, this accurate integrated navigation method can always keep high navigation accuracy. Figure 9 shows that although the navigation devices are out of order successively during the navigation process, compared with the system without fault, the attitude accuracy of this accurate integrated navigation method has almost no change. Particularly, there is no obvious change appearing in the attitude error curves. The heading accuracy is kept in the range of $\pm 1.6^{\prime}$, and pitch and roll accuracy are kept in the range of $\pm 0.9^{\prime}$. Figures 10 and 11 show that, because the navigation devices are out of order during the navigation process, there are some obvious changes appearing in the velocity and position error curves, but the velocity and position accuracy are still kept in the 
TABLE 1: Comparison of simulation results of SINS/GNSS/star sensor and SINS/GNSS-integrated navigation.

\begin{tabular}{|c|c|c|c|c|c|}
\hline $\begin{array}{l}\text { Integrated navigation } \\
\text { mode }\end{array}$ & $\begin{array}{l}\text { Heading } \\
\text { accuracy }\end{array}$ & $\begin{array}{c}\text { Horizontal attitude } \\
\text { accuracy }\end{array}$ & $\begin{array}{c}\text { Horizontal velocity } \\
\text { accuracy }\end{array}$ & $\begin{array}{c}\text { Vertical velocity } \\
\text { accuracy }\end{array}$ & $\begin{array}{l}\text { Position } \\
\text { accuracy }\end{array}$ \\
\hline SINS/GNSS/star sensor & $\pm 1.5^{\prime}$ & $\pm 0.9^{\prime}$ & $\pm 0.05 \mathrm{~m} / \mathrm{s}$ & $\pm 0.03 \mathrm{~m} / \mathrm{s}$ & $\pm 3.8 \mathrm{~m}$ \\
\hline SINS/GNSS & $\pm 4.1^{\prime}$ & $\pm 1.7^{\prime}$ & $\pm 0.11 \mathrm{~m} / \mathrm{s}$ & $\pm 0.06 \mathrm{~m} / \mathrm{s}$ & $\pm 4.5 \mathrm{~m}$ \\
\hline
\end{tabular}
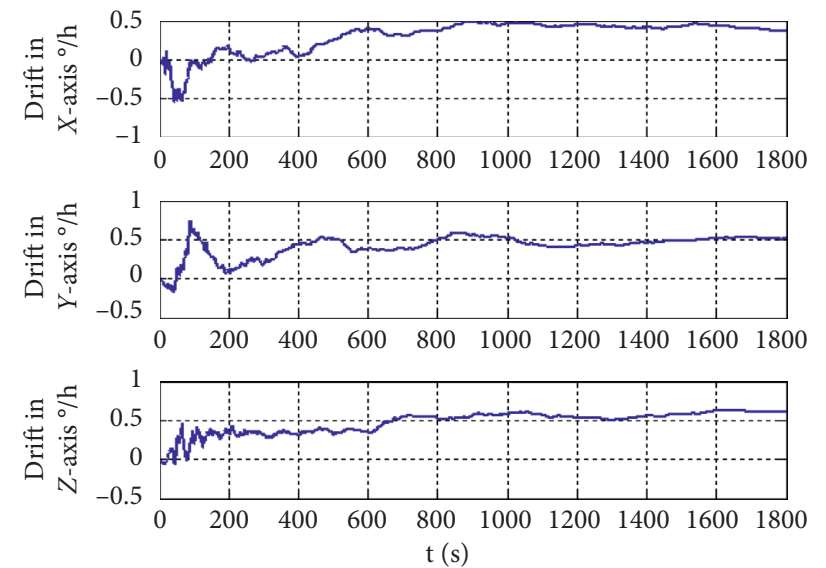

FIGURE 6: Estimating results on gyro constant drifts of accurate integrated navigation.
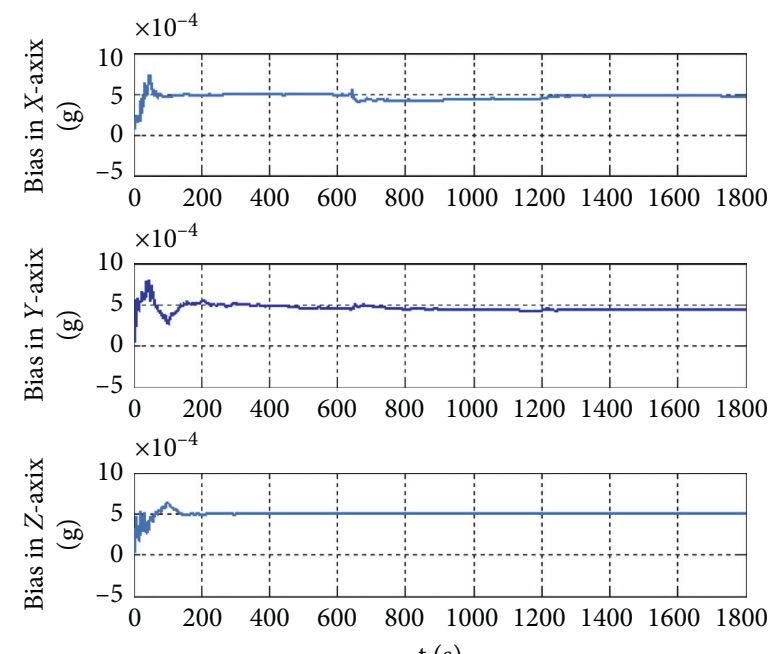

$\mathrm{t}(\mathrm{s})$

FIGURE 7: Estimating results on accelerometer constant biases of accurate integrated navigation.

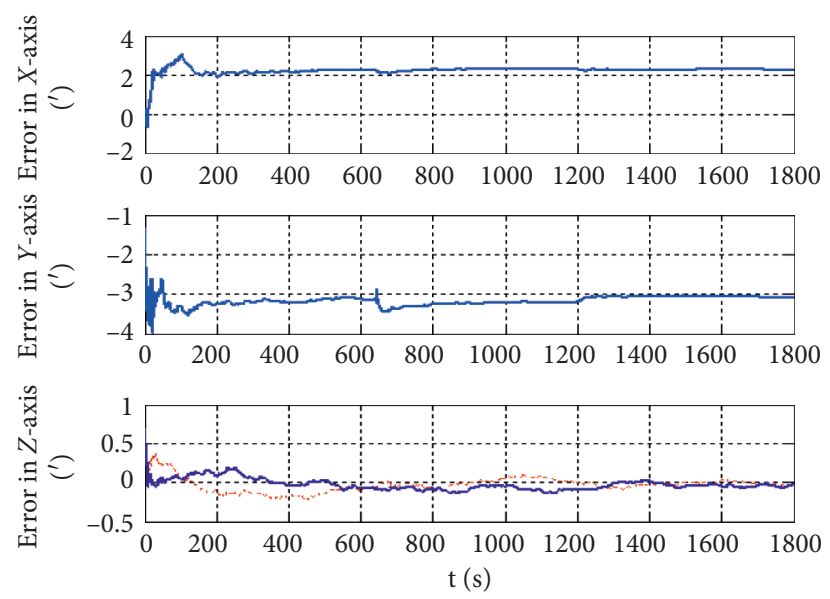

FIGURE 8: Estimating results on star sensor installation errors of accurate integrated navigation. 

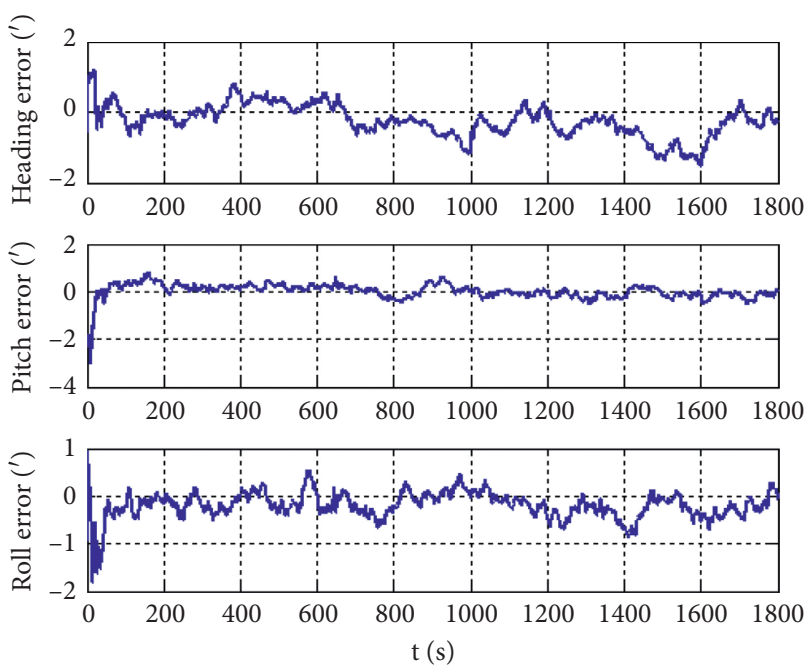

Figure 9: Attitude errors of accurate integrated navigation when system failure exists.
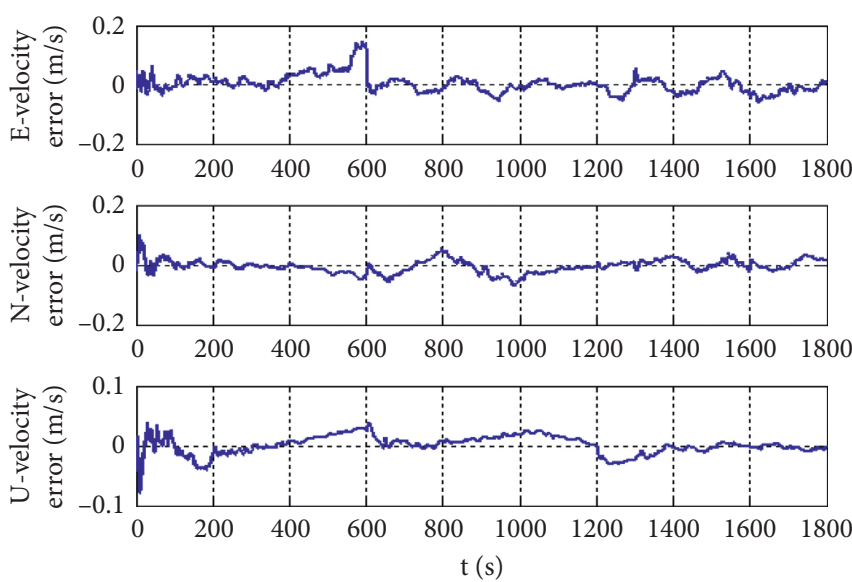

FIGURE 10: Velocity errors of accurate integrated navigation when system failure exists.
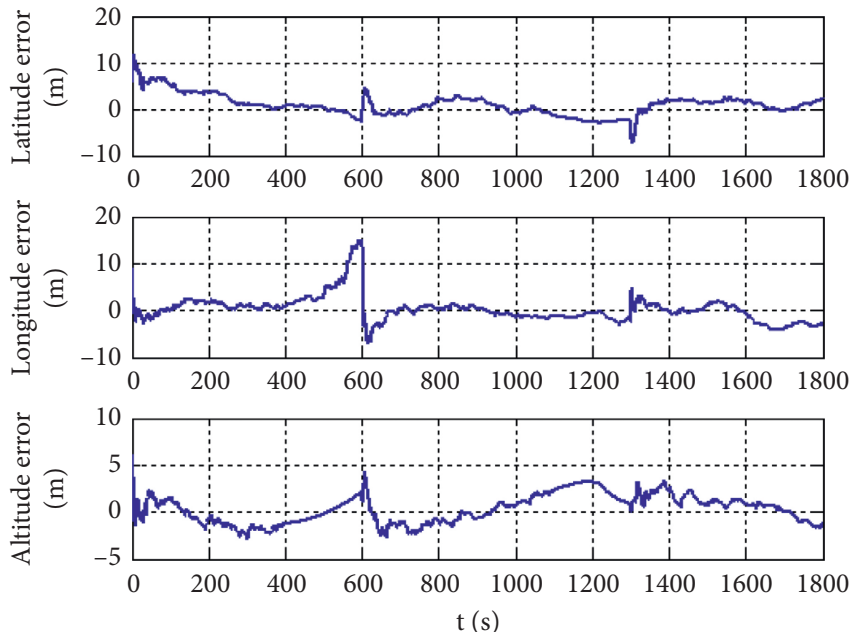

FIGURE 11: Position errors of accurate integrated navigation when system failure exists. range of $\pm 0.14 \mathrm{~m} / \mathrm{s}$ and $\pm 15.0 \mathrm{~m}$. Obviously, the velocity and position accuracy are not influenced badly by the failures of GNSS and star sensor. Therefore, according to the above simulation results, it is found that this accurate integrated navigation method has strong anti-interference capability, which can effectively solve the accurate navigation problem when GNSS fails to navigate momently for satellite signal being interfered with or shielded. In the same way, this accurate integrated navigation method also can realize the accurate navigation when star sensor is unable to output the attitude parameters for failing to search star. Consequently, these make this accurate integrated navigation method have strong survival capability in battlefield environment or harsh environment.

\section{Conclusions}

According to the above text, it can be seen that this accurate integrated navigation method not only fully absorbs the advantages of star sensor and GNSS but also achieves the higher attitude, velocity, and position accuracy by integrated navigation technology. And this accurate integrated navigation method can estimate errors of inertial instruments and installation errors of star sensor effectively. Therefore, this accurate integrated navigation method successfully overcomes the outstanding problems existing in SINS/ GNSS-integrated navigation, which are low heading accuracy and bad estimating effect on the constant drift of gyro in the $z$-axis. What is more, during the navigating process, when GNSS receiver or star sensor is unable to work momently, the outputs of SINS and another normal navigation device are used to construct measurements for integrated navigation filtering. Thus, it can be seen that this accurate integrated navigation method has strong anti-interference capability and survival capability in harsh environment.

Consequently, although the medium precision SINS and star sensor are used, the accurate integrated navigation method studied in this paper can still achieve high precision and high reliability integrated navigation, which is competent for the high accuracy navigation of high-altitude aerocrafts, and it has a good prospect of engineering application.

\section{Data Availability}

The simulation experiment data used to support the findings of this study are available from the corresponding author (Yang Xiaogang; doctoryxg@163.com) upon request.

\section{Conflicts of Interest}

The authors declare that there are no conflicts of interest regarding the publication of this paper.

\section{Acknowledgments}

This paper and related research work are funded by the Natural Science Foundation of Shaanxi Province (Grant no. 2020JM-355). 


\section{References}

[1] E. S. Abdolkarimi and M. R. Mosavi, "Wavelet-adaptive neural subtractive clustering fuzzy inference system to enhance low-cost and high-speed INS/GPS navigation system," GPS Solutions, vol. 24, no. 2, pp. 1536-1551, 2020.

[2] G. Hu, L. Ni, B. Gao, X. Zhu, W. Wang, and Y. Zhong, "Model predictive based unscented kalman filter for hypersonic vehicle navigation with INS/GNSS integration," IEEE Access, vol. 8, pp. 4814-4823, 2020.

[3] Y. Liu, X. Fan, C. Lv, J. Wu, L. Li, and D. Ding, "An innovative information fusion method with adaptive kalman filter for integrated INS/GPS navigation of autonomous vehicles," Mechanical Systems and Signal Processing, vol. 100, no. 1, pp. 605-616, 2018.

[4] G. Hu, B. Gao, Y. Zhong, and C. Gu, "Unscented kalman filter with process noise covariance estimation for vehicular INS/ GPS integration system," Information Fusion, vol. 64, pp. 194-204, 2020.

[5] YuexinL. Zhang et al., "A hybrid intelligent algorithm DGPMLP for GNSS/INS integration during GNSS outages," Journal of Navigation, vol. 72, no. 2, pp. 375-388, 2019.

[6] G. Hu, S. Gao, and Y. Zhong, "A derivative UKF for tightly coupled INS/GPS integrated navigation," ISA Transactions, vol. 56, pp. 135-144, 2015.

[7] Bo Yang, B. Shan, Y. Wang et al., "MIMU/GPS precise integrated navigation method aided by heading information," Journal of Chinese Inertial Technology, vol. 26, no. 5, pp. 89-94, 2018.

[8] M. Z. Mostafa, H. A. Khater, M. R. Rizk et al., "A novel GPS/ RAVO/MEMS-INS smartphone sensors integrated method to enhance USV navigation system during GPS outages," Measurement Science and Technology, vol. 30, no. 9, Article ID 095103, 2019.

[9] Q. Wang, M. Diao, W. Gao, M. Zhu, and S. Xiao, "Integrated navigation method of a marine strapdown inertial navigation system using a star sensor," Measurement Science and Technology, vol. 26, no. 11, p. 115101, 2015.

[10] X. Ning, J. Zhang, M. Gui, and J. Fang, "A fast calibration method of the star sensor installation error based on observability analysis for the tightly coupled SINS/CNS-integrated navigation system," IEEE Sensors Journal, vol. 18, no. 16, pp. 6794-6803, 2018.

[11] G. Zhaohui, M. Dejun, Z. Yongmin et al., "A strap-down inertial navigation/spectrum red-shift/star sensor (SINS/SRS/ SS) autonomous integrated system for spacecraft navigation," Sensors, vol. 18, no. 7, pp. 2039-2055, 2018.

[12] B. Gao, G. Hu, S. Gao, Y. Zhong, and C. Gu, "Multi-sensor optimal data fusion for INS/GNSS/CNS integration based on unscented kalman filter," International Journal of Control, Automation and Systems, vol. 16, no. 1, pp. 129-140, 2018.

[13] R. Wang, Z. Xiong, J. Liu et al., "SINS/GPS/CNS Information fusion system based on improved huber filter with classified adaptive factors for high-speed UAVs," IEEE, in Proceedings of IEEE PLANS, Position Location and Navigation Symposium, pp. 441-446, IEEE, Myrtle Beach, SC, USA, April 2012.

[14] Bo Yang, Y.-gang Wang, and H.-tao Xu, "High-precision integrated inertial/satellite/starlight navigation for missile," Journal of Chinese Inertial Technology, vol. 18, no. 4, pp. 444-449, 2010.

[15] Y.-ling Min, Z. Xiong, Li Xing et al., "An improved SINS/ GNSS/CNS federal filter based on dual quaternions," Acta Armamentarii, vol. 39, no. 2, pp. 315-324, 2018.
[16] G. Hu, W. Wang, Y. Zhong, B. Gao, and C. Gu, "A new direct filtering approach to INS/GNSS integration," Aerospace Science and Technology, vol. 77, pp. 755-764, 2018.

[17] H. Zhang, Y. Niu, J. Lu et al., "Star sensor installation error calibration in stellar-inertial navigation system with a regularized backpropagation neural network," Measurement Science and Technology, vol. 29, no. 8, Article ID 085102, 2018. 